Mark Angelo C. Ang, MD'

Ariel M. Vergel De Dios, MD²

Jose M. Carnate, Jr. MD²

'Department of Laboratories

Philippine General Hospital

University of the Philippines Manila

${ }^{2}$ Department of Pathology

College of Medicine

University of the Philippines Manila
Correspondence: Dr. Mark Angelo C. Ang

Department of Laboratories

Philippine General Hospital

University of the Philippines Manila

Taft Avenue, Ermita, Manila 1000

Philippines

Phone (632) 5548400 local 3208

Email: mark.ang.2010@gmail.com

Reprints will not be available from the author.

The authors declared that this represents original material that is not being considered for publication or has not been published or accepted for publication elsewhere, in full or in part, in print or electronic media; that the manuscript has been read and approved by all authors, that the requirements for authorship have been met by each author, and that the authors believes that the manuscript represents honest work.

Disclosures: The authors signed a disclosure that there are no financial or other (including personal) relationships, intellectual passion, political or religious beliefs, and institutional affiliations that might lead to a conflict of interest.

\section{Primary Sinonasal Ameloblastoma in a Filipino Female}

\begin{abstract}
Primary sinonasal ameloblastoma is an extremely rare odontogenic epithelial tumor histomorphologically identical to its gnathic counterparts but with distinct epidemiologic and clinicopathologic characteristics. We present a case of a 46-year-old female with a one year history of recurrent epistaxis, nasal obstruction and frontonasal headache. Clinical examination, CT scan and subsequent surgical excsion revealed an intranasal mass attached to the lateral nasal cavity with histomorphologic features of ameloblastoma and was signed out as extragnathic soft tissue ameloblastoma of the sinonasal area. Extraosseous extragnathic primary sinonasal ameloblastoma are rare but do occur and should be distinguished from infrasellar craniopharyngiomas.
\end{abstract}

Keywords: extraosseous, extragnathic, sinonasal, ameloblastoma

Ameloblastomas are slow growing locally aggressive odontogenic epithelial tumors of the jaw and are classified into solid/multicystic, unicystic, desmoplastic, and peripheral subtypes. ${ }^{1,2,3}$ They involve the mandible $80 \%$ of the time and are often associated with an unerrupted molar tooth. Extraosseous extragnathic Ameloblastomas are very rare, occurring less than 1.3 to $10 \%$ of all ameloblastomas with all cases reported so far arising from the sinonasal region. ${ }^{1,2,4}$ We present a case of primary sinonasal ameloblastoma in a Filipino female.

\section{CASE REPORT}

A 46-year-old female consulted at the University of the Philippines - Philippine General Hospital Department of Otorhinolayngology with a one-year history of recurrent, spontaneous epistaxis from the right nose, associated with ipsilateral nasal obstruction, thin-brown rhinorrhea, and frontonasal headache relieved by oral paracetamol. Nasal endoscopy revealed a pale pink irregularly shaped polypoid mass attached to the lateral nasal wall almost completely obstructing the nasal cavity. Plain coronal and sagittal CT images of the nasal cavity and paranasal sinuses showed opacification of the right nasal chamber by soft tissue densities with obstruction of the ipsilateral ostiomeatal unit and sphenoethmoidal recess (Figure 1). The sphenoid, frontal and contralateral paranasal sinuses and nasal vault were uninvolved. Incision biopsy was read as sinonasal exophytic papilloma and the mass was excised via endoscopic sinus surgery under general anesthesia. 


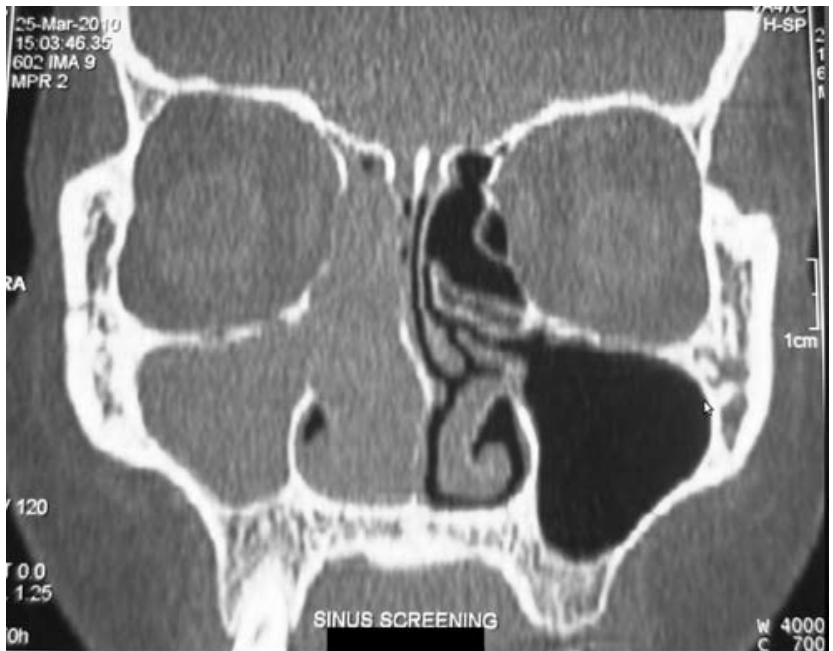

Figure 1. Computed tomography scan of the head, plain, coronal cut at the level of the nasal cavity and paranasal sinuses. There is opacification of the right nasal chamber by soft tissue densities with obstruction of the ostiomeatal unit and sphenoethmoidal recess.

The submitted specimen consisted of a $2 \mathrm{~cm}$ by $0.8 \mathrm{~cm}$ cream white solid, soft to rubbery mass. On histologic examination, trabecula and islands of cytologically benign odontogenic epithelium permeate an edematous, myxoid, hypocellular stroma. Columnar cells that display palisading and reverse polarity, line the periphery of the epithelium.
At the center of the epithelial islands, loose collections of stellate and spindly cells similar to the stellate reticulum of the embryonic enamel organ are found. Acanthomatous changes are present in the superficial layers. There is no atypia and no mitosis (Figures 2 and 3). This case was signed out as extragnathic soft tissue ameloblastoma.

\section{DISCUSSION}

Most reported cases of ameloblastoma in the sinonasal cavity actually describe tumors that originated from the maxilla and have only secondarily involved the sinonasal area. ${ }^{4}$ To date, the 26-year review by Schafer et al. of 24 primary sinonasal tract ameloblastomas at the Armed Forces Institute of Pathology remains the single largest series describing this entity. ${ }^{4}$ Although three additional case reports were recently published, to the best of our knowledge, this is the first case of primary sinonasal ameloblastoma in the Philippines. ${ }^{5,6,7}$ Unlike our patient, primary sinonasal ameloblastomas more commonly affect males with mean age at presentation of 59.7 years. ${ }^{1.4}$ Patients usually present with an intranasal mass, nasal obstruction, sinusitis and epistaxis of one month to several years duration. ${ }^{1,4}$ Radiologically, sinonasal ameloblastomas are solid masses or opacifications rather than multilocular and radiolucent as those that arise within the jaws. ${ }^{1}$

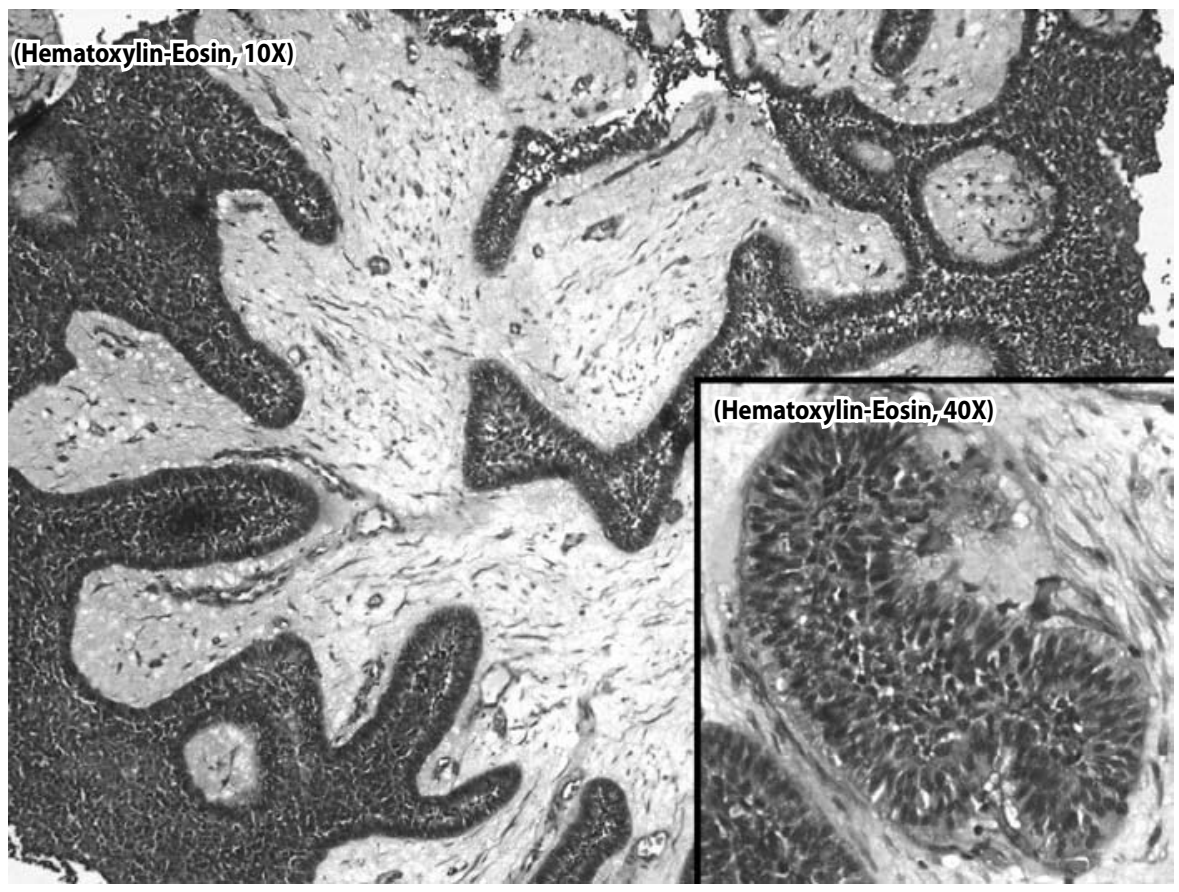

Figure 3. Image from another area showing the same epithelial trabecula and the edematous, myxoid stroma (Hematoxylin and Eosin, 10x). Inset: A typical epithelial island with the classic reverse polarization of the peripheral columnar cells (Hematoxylin and Eosin, 40x). 


\section{UNDER THE MICROSCOPE}

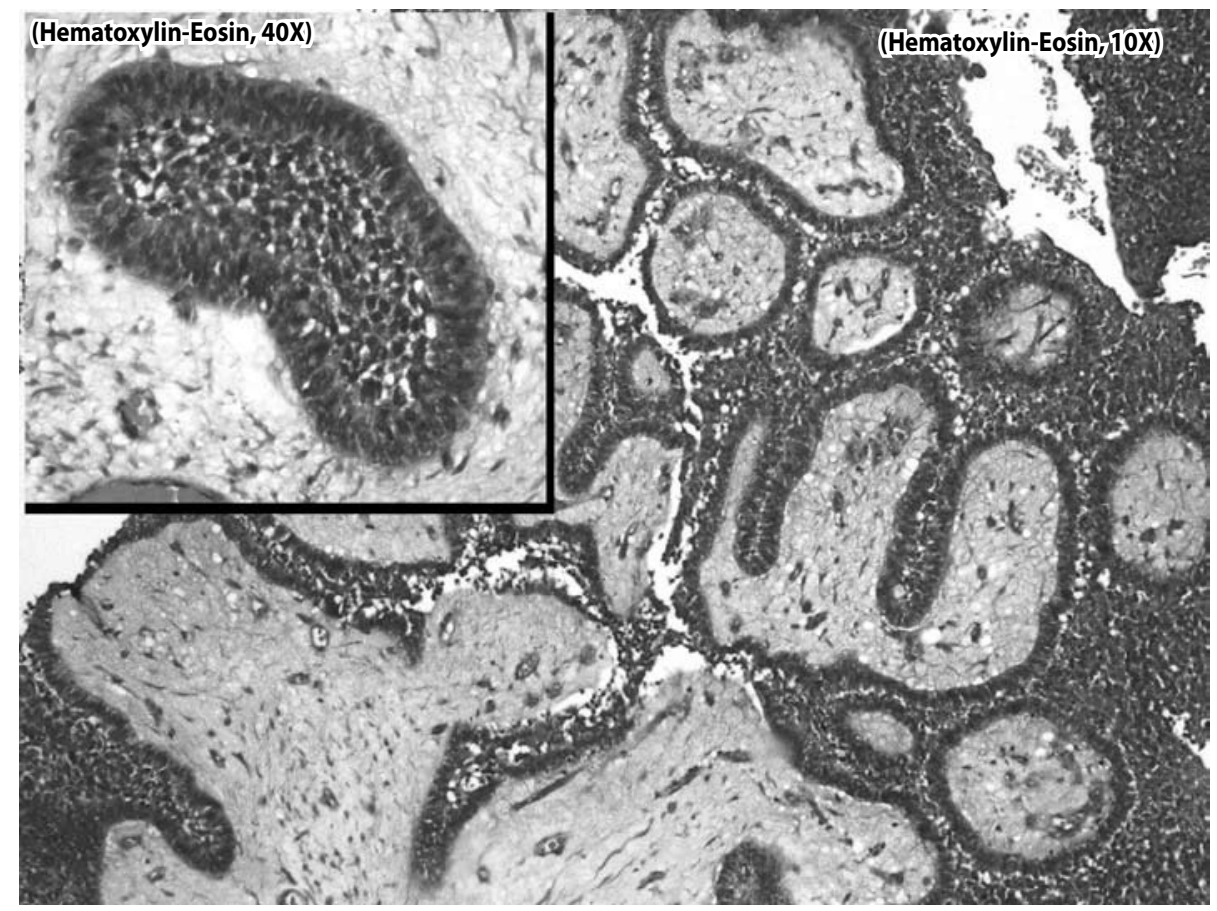

Figure 2. Interconnecting trabecula and islands of benign odontogenic epithelium in an edematous, myxoid, hypocellular stroma (Hematoxylin and Eosin, 10x). Inset: An epithelial island with peripheral columnar cells that display palisading and reverse polarity. The center shows a loose collection of stellate and spindly cells, similar to the stellate reticulum of the embryonic enamel organ (Hematoxylin and Eosin, 40x).

The histomorphologic features of primary sinonasal ameloblastomas are identical to their gnathic counterparts and include unencapsulated proliferating nests, islands or sheets of odontogenic epithelium resembling the embryonic enamel organ. The epithelium is composed of a central area of loosely arranged cells similar to the stellate reticulum of the enamel organ and a peripheral layer of palisading columnar or cuboidal cells with hyperchromatic small nuclei oriented away from the basement membrane, the so called reverse polarity.' Experts believe that primary sinonasal ameloblastomas arise from remnants of odontogenic epithelium, lining of odontogenic cysts, basal layer of the overlying oral mucosa or heterotopic embryonic organ epithelium. ${ }^{1,4}$ This is supported by the observation that the ameloblastomatous epithelial proliferations are often seen in continuity with native sinonasal (schneiderian) epithelium. ${ }^{1,4}$

This entity should be distinguished from an infrasellar craniopharyngioma which is an important differential diagnosis that is often difficult and often virtually impossible to differentiate from a primary sinonasal ameloblastoma solely on histomorphologic grounds. In most cases, however, clinicopathologic correlation guides the diagnosis ${ }^{8}$ and special stains are of limited utility.

Surgical excision is the treatment of choice, the type and extent of which is dictated by the size and localization of the lesion. Recurrence can occur, generally within two years but overall treatment success depends on complete surgical eradication. No deaths, metastases or malignant transformation have so far been reported ${ }^{1,4}$ and our patient is free of disease, 15 months post surgery.

\footnotetext{
REFERENCES

1. Wenig BM. "Sinonasal ameloblastoma." Atlas of Head and Neck Pathology. 2nd ed. Philadelphia: Elsevier Saunders; 2008. 95-97.

2. International Agency for Research on Cancer. Pathology and Genetics of Head and Neck Tumours. Lyon: IARC Press; 2005.

3. Gnepp DR. Diagnostic Surgical Pathology of the Head and Neck. 2nd ed. Philadelphia, Pa: Saunders; 2009.

4. Schafer DR, Thompson LDR, Smith BC, Wenig BM. Primary ameloblastoma of the sinonasal tract. Cancer 1998 Feb; 82(4):667-674.

5. Leong SC, Karkos PD, Krajacevic J, Islam R, Kent SE. Ameloblastoma of the sinonasal tract: A case report. Ear Nose Throat J 2010 Feb;89(2):70-71.

6. Ereño C, Etxegarai L, Corral M, Basurko JM, Bilbao FJ, López Jl. Primary sinonasal ameloblastoma. APMIS 2005 Feb;113(2):148-150.

7. Guilemany JM, Ballesteros F, Alós L, Alobid I, Prades E, Menéndez LM, Cardesa A. Plexiform ameloblastoma presenting as a sinonasal tumor. Eur Arch Otorhinolaryngol 2004 Jul;261(6):304306.

8. Deutsch H, Kothbauer K, Persky M, Epstein FJ, Jallo GI. Infrasellar craniopharyngiomas: case report and review of the literature. Skull Base 2001 May;11(2):121-128.
} 